the sustainability of older women's wellness initiatives. The forum was a collaborative project of the Department of Ageing and Disability, NSW Department of Health, Department for Women, OWN and the Benevolent Society for the International Year of Older Persons. Projects showcased at the forum included those operating in a remote rural community in the New England area and partnership models between groups of older women and local councils or community health services. A key component of successful wellness initiatives is ownership of the project by older women.

As part of the NSW Healthy Ageing Framework, funding from the National Women's Health Program has been allocated to two projects to promote older women's wellness. The Rural Women's Network has received funding to implement a multimedia campaign for rural older women, with particular emphasis on women in remote areas. OWN has received funding to build on the outcomes of the Older Women's Wellness Forum and to promote older women's wellness models.

\section{REFERENCES}

1. What the figures show: Older women in Australia. Sydney: Older Women's Network (Australia) Inc, 1997.

2. NSW Department of Health. Chief Health Officer's Report 1997. Sydney: NSW Department of Health, 1997.

3. A picture of wellness: The story of the Bankstown Older Women's Wellness Centre. Sydney: Older Women's Network (Australia) Inc, 1997. $\mathrm{F}$

\title{
FROM COMMUNITY ATTITUDES TO COMMUNITY ACTION: OPPOSING DOMESTIC VIOLENCE
}

\author{
Margo Moore \\ Women's Health Coordinator \\ Division of Population Health \\ South Western Sydney Area Health Service

\section{Anne Connolly} \\ Women's Health Coordinator \\ Division of Population Health \\ Central Sydney Area Health Service
}

This article reports on the results of a survey to evaluate the effectiveness of an intervention to raise awareness about domestic violence within four community groups: Arabic, Tongan, Vietnamese and Chinese, in the South Western and Central Sydney Area.

The 1996 Women's Safety Australia survey, conducted by the Australian Bureau of Statistics, established a population prevalence of domestic violence for Australian women. ${ }^{1}$ Of women who had ever been married or lived in a de facto relationship, 22.5 per cent had experienced violence by a partner at some time during the relationship. Sixty-one percent of women who experienced violence by a current partner reported having children in their care and 36 per cent reported that their children had witnessed the violence.

The high prevalence of domestic violence occurs alongside a high tolerance to domestic violence within the Australian community. A survey of community attitudes to domestic violence by the Office of the Status of Women in 1995 found that 18 per cent of Australians believe there are circumstances in which domestic violence is acceptable. ${ }^{2}$ Despite what is now known about the prevalence of and attitudes to domestic violence in the general community, less is known about attitudes to domestic violence in specific cultural groups in Australia.

A recent study measured attitudes to domestic violence in Arabic, Tongan, Vietnamese and Chinese community groups before and after implementation of a communitybased intervention to change attitudes towards, as well as raise awareness about, domestic violence. ${ }^{3}$ The study compared differences between the four groups and the results of the 1995 national survey on attitudes to domestic violence in the Australian community.

The results showed significant differences in attitudes to domestic violence between the four cultural groups and the larger Australian community. Pre-intervention respondents in the four groups were less informed about domestic violence and had less serious perceptions of the types of violence that occur. Following a media campaign and a number of community events, the second survey showed significant changes in attitudes and awareness about domestic violence. This result was more pronounced in the new arrival groups. In the preintervention survey, domestic violence was more commonly seen as an issue to be dealt with by the family, an attitude that changed significantly after the intervention. After the campaign and community events, people were more likely to identify domestic violence as an issue, and were more likely to rate the types of domestic violence as serious. In the post-intervention survey, 77 per cent of respondents rated domestic violence as a crime, compared to 63 per cent in the pre-intervention survey.

Successful strategies to address domestic violence involve intersectoral responses by the police, legal, community and government agencies. However, most non-English 
speaking background community groups have not been involved in these coordinated responses to domestic violence.This research established the need to work with specific cultural groups to address domestic violence. It successfully challenged the tolerance to domestic violence and showed that community involvement is crucial in designing culture-specific interventions. The study aimed to strengthen existing community capacity to address domestic violence within each community group by building on existing networks. The project was a balanced approach to providing communities with information and skills about how to respond to domestic violence and recognising and building on existing community strengths.

\section{REFERENCES}

1. Australian Bureau of Statistics (1996) Women's safety Australia (Cat. No. 4128.0). Canberra: Commonwealth of Australia, 1996.

2. Office of the Status of Women. Community attitudes to violence against women. Canberra: Department of the Prime Minister and Cabinet, 1995.

3. Strengthening attitudes opposing domestic violence in culturally diverse communities-Campaign report. 1998. Sydney: South Western and Central Sydney Area Health Services, 1998. ISBN 1875909 540. it?

\section{SMOKING AND WOMEN}

\section{Kate Purcell}

Principal Policy Officer

Public Health Legislation Project

NSW Department of Health

This article provides an overview of the effect of smoking on women and how health services can support women to quit.

For almost 60 years, until the recent restrictions on advertising, women have been exposed to images of smoking in a variety of media, including billboard advertisements, movies and women's magazines. Tobacco company documents confirm that the industry has spent large sums of money positioning smoking as a gateway to glamour, beauty, independence, a slim body, success and romance. In Australia, the industry aggressively marketed brands designed to appeal to teenage girls and young women.

In 1992, just over 5,000 Australian women died from tobacco-related illness (more than one woman every two hours), and lung cancer is second only to breast cancer as the most common cause of cancer deaths among women. Recent research indicates that women who smoke at the same level as men have a higher risk of developing lung cancer than men. ${ }^{1}$ Other research suggests that the younger people are when they take up smoking, the greater their chance of developing cancer later in life because early smoking appears to cause greater damage to DNA. ${ }^{2}$

Like men, women who smoke are likely to die of lung and other cancers, heart disease, stroke and respiratory diseases. In addition, women also experience genderspecific problems and additional health risks. These include:
- Menstrual problems and reduced fertility

Women who smoke more than 20 cigarettes a day are three times more likely than non-smokers to take more than a year to conceive and have a greater risk of ectopic pregnancy and miscarriage. ${ }^{3}$

- Increased risk of cancer of the cervix and vulva It has been estimated that 19 per cent of cervical cancer and 40 per cent of vulvar cancer is caused by smoking. ${ }^{4}$

- Increased risk of osteoporosis

For women who smoke 20 cigarettes a day through adulthood, bone density will have reduced by five to 10 per cent by the time they reach menopause. ${ }^{4}$

- Prenatal problems

Smoking women experience complications in pregnancy and labour, including a greater risk of miscarriage, premature labour and low-birth-weight babies. $^{4}$

\section{- Postnatal problems}

Children of smoking mothers are at increased risk of SIDS and childhood asthma. ${ }^{4}$

- Heart disease and stroke

Women who use oral contraceptives and who smoke have a tenfold increase in risk of heart disease and stroke compared to non-smokers. ${ }^{5}$

Health professionals have an important role in providing information and in encouraging and supporting women in their attempts to quit smoking. About 180,000 Australians quit smoking each year and, for most smokers, successful quitting usually takes a number of attempts.

After quitting, the body can rid itself of nicotine and carbon monoxide within a few hours and nicotine byproducts within a few days. Blood flow to the limbs improves within two months, and the lungs clean 